\title{
Effets d'une tuyère convergente sur un écoulement tournant
}

\author{
Benjamin Leclaire ${ }^{a}$, Laurent Jacquin et Denis Sipp \\ ONERA/DAFE, 8 rue des Vertugadins, 92190 Meudon, France
}

Reçu le 25 février 2005, accepté le 21 mars 2005

\begin{abstract}
Résumé - Nous étudions l'écoulement dans le plan de sortie d'un jet tournant à grand nombre de Reynolds généré à l'aide d'un nid d'abeilles tournant suivi d'une tuyère convergente. Des mesures fil chaud réalisées en sortie de deux tuyères de taux de contraction différents montrent l'apparition progressive de turbulence pour des valeurs identiques du taux de rotation de l'écoulement en amont des tuyères. Afin d'expliquer ce phénomène, nous étudions donc la dynamique de l'écoulement de conduite compris entre le nid d'abeilles et le plan de sortie des convergents. Un modèle stationnaire simple d'écoulement est d'abord introduit. Il montre la possibilité d'une recirculation à la paroi de la tuyère lorsque les conditions amont sont proches de la criticité, définie au sens des ondes inertielles pouvant se propager dans l'écoulement. Nous trouvons ensuite que ces solutions sont globalement stables à des perturbations axisymétriques, mais sont susceptibles d'amplifier fortement des perturbations de petite échelle initiées à l'amont de l'écoulement et suivies le long de leur trajectoire jusqu'en sortie.
\end{abstract}

Mots clés : Écoulement tournant / transition critique / recirculation / instabilité

\begin{abstract}
Effects of a converging nozzle on a rotating flow. We study the flow in the exit plane of a high-Reynolds swirling jet generated with a rotating honeycomb followed by a converging nozzle. Hot-wire measurements downstream of two nozzles of different contraction ratios show that the flow progressively becomes turbulent for the same values of the swirl number characterising the flow upstream of the nozzles. To understand this phenomenon, we investigate the pipe flow located between the exit of the honeycomb and the exit plane of the nozzles. A simple stationary model is first introduced, which shows that a recirculation is likely to occur at the nozzle wall when the upstream flow is critical with respect to inertial waves. We then show that these solutions are globally stable to axisymmetric disturbances, but may strongly enhance short-scale perturbations on their trajectory from the outlet of the honeycomb to the exit plane of the nozzles.
\end{abstract}

Key words: Swirling flow / criticality / recirculation / instability

\section{Introduction}

Le problème de l'interaction d'un écoulement tournant avec une tuyère convergente se pose dans le domaine des turbomachines et celui des systèmes de combustion industriels, mais aussi dans le cadre plus théorique des expériences de jet tournant. En effet, dans ces dernières, un convergent final est souvent utilisé pour générer un écoulement de sortie régulier et peu turbulent, qui est pris comme condition génératrice du jet développé. Dans la plupart des travaux existants, l'attention se porte alors sur l'évolution du profil de vitesse dans le jet [1] ou encore sur l'apparition éventuelle, suivant les régimes, d'instabilités centrifuges ou d'une bulle d'éclatement tourbillonnaire [2]. Ces deux phénomènes en particulier sont encore

\footnotetext{
a Auteur correspondant : leclaire@onera.fr
}

partiellement incompris et rendent la prévision théorique et numérique des écoulements tournants particulièrement délicate.

En revanche, peu de travaux sur le jet tournant ont étudié l'écoulement en amont de son plan de sortie. Dans cette étude, nous souhaitons comprendre quelle est l'influence de la tuyère convergente sur les propriétés de l'écoulement obtenu dans ce plan. Nos mesures montrent en effet qu'à forte rotation celui-ci devient progressivement turbulent, et que l'origine de ce phénomène doit être vraisemblablement recherchée en amont de ce plan.

Nous présenterons donc d'abord le protocole expérimental retenu ainsi que les résultats des mesures effectuées dans le plan de sortie de jets générés par deux tuyères convergentes différentes. Nous nous intéresserons ensuite aux caractéristiques de l'écoulement se développant depuis la section aval du système de mise 
en rotation jusqu'au plan de sortie de la tuyère. Pour cela, nous étudierons d'abord un modèle stationnaire simplifié, avant d'en dégager les principales caractéristiques de stabilité. Enfin, ces résultats seront confrontés à ceux obtenus expérimentalement, afin de comprendre les limites du modèle.

\section{Données expérimentales}

\subsection{Dispositif et protocole}

La soufflerie R4Ch de l'ONERA a pour but de générer des écoulements tournants comportant une large zone de rotation solide. Elle est munie d'une portion de veine rotative équipée d'un nid d'abeilles à mailles très fines (de l'ordre de 1,5 mm de diamètre) permettant de superposer à un écoulement axial initial (supposé en première approximation uniforme, de vitesse $\left.U_{0}\right)$ un mouvement de rotation solide de vitesse angulaire $\Omega_{0}$. L'écoulement se développe ensuite dans une portion de conduite de section constante avant d'être accéléré par une tuyère convergente (voir la Fig. 1).

Dans le plan de sortie, l'écoulement est mesuré suivant un rayon à l'aide d'une sonde fil chaud deux composantes, ce qui permet de connaître les composantes axiale et azimutale instantanées de la vitesse (dans toute cette étude, nous raisonnerons dans un système de coordonnées cylindrique, l'axe étant confondu avec celui de la veine). Un traitement du signal adéquat permet ensuite de reconstituer les valeurs moyennes ainsi que les taux de turbulence.

Lors d'une série d'essais, la vitesse axiale amont $U_{0}$ est initialement réglée par une mesure de la vitesse au centre du plan de sortie de la tuyère convergente. La vitesse de rotation du nid d'abeilles $\Omega_{0}$ est ensuite augmentée par paliers et un profil de vitesse est réalisé à chacun d'entre eux. Les essais présentés ici correspondent tous à une vitesse $U_{0}=3 \mathrm{~m} . \mathrm{s}^{-1}$, et la vitesse de rotation maximale du nid est égale à 600 tr. $\mathrm{min}^{-1}$.

\subsection{Paramètres de contrôle}

La dynamique des écoulements tournants est habituellement caractérisée par le nombre de Reynolds et le nombre de Swirl. Ce dernier caractérise l'importance de la vitesse de rotation par rapport à la vitesse axiale, et est en particulier utilisé pour prévoir l'apparition d'instabilités centrifuges ou d'un éclatement tourbillonnaire (voir par exemple [2]).

Dans notre écoulement, la présence d'une tuyère convergente introduit des fortes variations de l'écoulement dans la direction axiale, si bien que les nombres de Reynolds et de Swirl n'y sont pas constants. Nous raisonnerons donc dans un premier temps sur les paramètres concernant l'écoulement en amont de la tuyère (indicé par 0 ). Ainsi, les réglages de $U_{0}$ et $\Omega_{0}$ mentionnés précédemment nous donnent comme valeurs typiques :

$$
R e_{0}=\frac{R_{0} U_{0}}{\nu} \approx 30000
$$

et

$$
S_{0}=\frac{R_{0} \Omega_{0}}{U_{0}} \approx 0 \quad \text { à } 3
$$

Par ailleurs, nous utilisons deux tuyères convergentes de taux de contraction égaux à 4 et 9 . Cette grandeur est définie comme le rapport entre les aires à l'amont et à l'aval de la tuyère :

$$
\chi=\left(\frac{R_{0}}{R_{1}}\right)^{2}
$$

(voir les notations sur la Fig. 1).

\section{3 Écoulement en sortie de tuyère}

\subsubsection{Champ moyen}

La figure 2 présente à titre d'exemple les profils de vitesse obtenus en sortie du convergent $\chi=9$. Lorsque $S_{0}$ augmente, la vitesse axiale n'est plus uniforme, mais adopte un profil parabolique : cet effet de création de vorticité azimutale par interaction entre vorticité axiale et convergence est connu et a été analysé notamment par Batchelor [3]. Par ailleurs, on constate que l'écoulement possède une large zone de rotation solide, et que celle-ci diminue lorsque la rotation amont augmente. On observe un comportement comparable en sortie du convergent $\chi=4$.

En notant $U_{1}=U_{z}\left(S_{0}=0\right)$ la vitesse axiale moyenne de l'écoulement obtenu au centre du plan de sortie de la tuyère en l'absence de rotation, on définira donc :

$$
R e_{1}=\frac{R_{1} U_{1}}{\nu} \approx \sqrt{\chi} R e_{0}
$$

et

$$
S_{1}=\frac{R_{1} \Omega_{1}}{U_{1}}
$$

où $\Omega_{1}$ désigne la vitesse angulaire au centre du plan de sortie. On peut montrer qu'on a alors de façon approchée : $S_{1} \approx S_{0} / \sqrt{\chi}$. En particulier, il est important de noter que les écoulements de sortie obtenus pour des écoulements amont identiques (même $S_{0}$ ) mais correspondant à des tuyères différentes n'ont pas le même nombre de Swirl de sortie, donc des propriétés dynamiques locales différentes.

\subsection{2 Énergie cinétique turbulente}

Les taux de turbulence dans la direction axiale et azimutale sont définis par

$$
q_{z}=\frac{\sqrt{\sigma_{z}}}{U_{1}} \quad \text { et } \quad q_{\theta}=\frac{\sqrt{\sigma_{\theta}}}{U_{1}}
$$

où $\sigma$ désigne l'écart-type de chaque composante. Il ne s'agit donc pas d'un taux de turbulence local puisqu'on prend systématiquement $U_{1}$ comme vitesse moyenne de référence. 

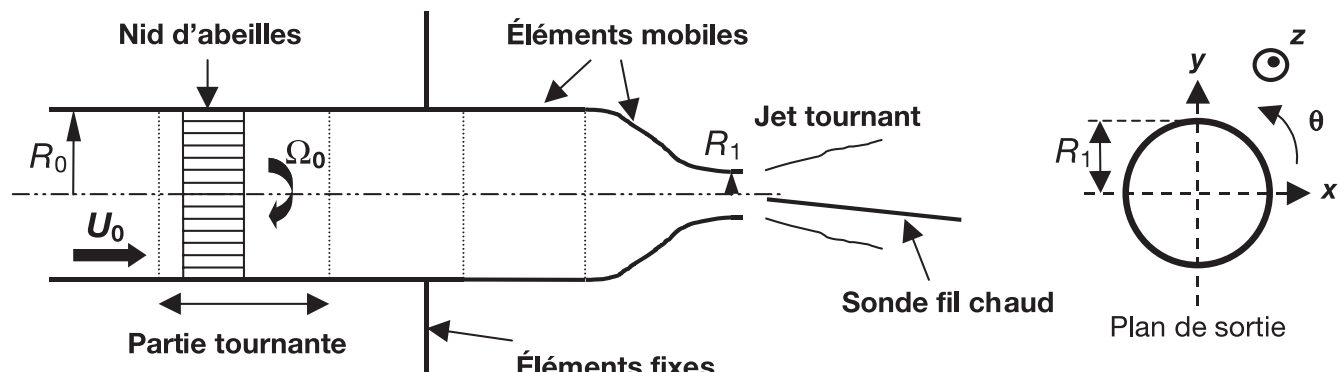

Fig. 1. Dispositif expérimental et localisation des mesures.

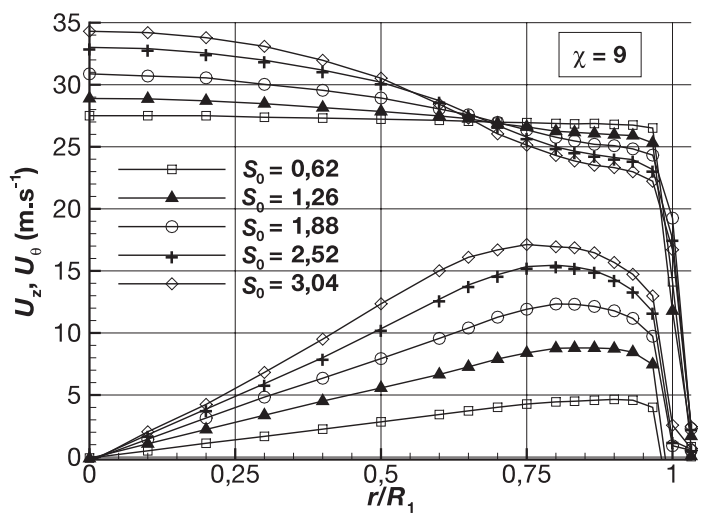

Fig. 2. Composantes axiale et azimutale de la vitesse suivant un rayon du plan de sortie du convergent $\chi=9$, pour différents nombres de Swirl $S_{0}$.

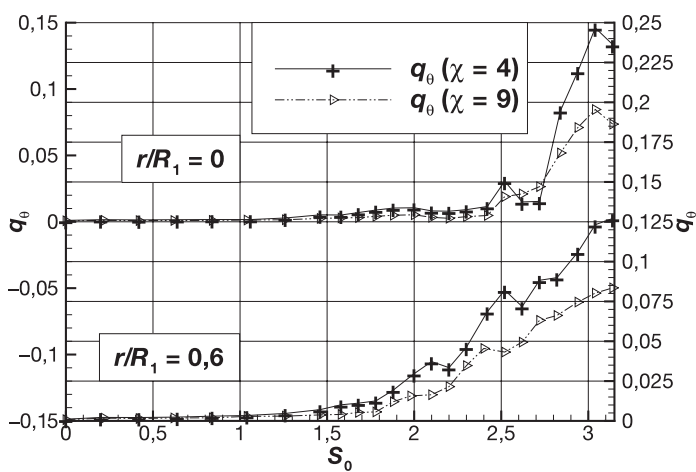

Fig. 3. Évolution de $q_{\theta}$ en fonction de $S_{0}$ au centre, et au rayon adimensionné $r / R_{1}=0,6$, pour les deux tuyères convergentes.

Lorsque la rotation amont augmente, on observe pour les deux tuyères que les taux de turbulence augmentent progressivement, d'abord à la périphérie puis dans tout le plan de sortie. La figure 3 montre que cette évolution est plutôt contrôlée par le nombre de Swirl amont $S_{0}$ que par celui caractérisant l'écoulement du plan de sortie, $S_{1}$. C'est précisément pour cette raison qu'il nous apparaît nécessaire de comprendre la dynamique de l'écoulement de conduite en amont de ce plan.

\section{Modèle de fluide parfait stationnaire}

\subsection{Formulation du problème}

Nous utilisons ici le modèle décrit par Batchelor [3], déjà évoqué à la section précédente. Dans le cas d'un écoulement axisymétrique incompressible de fluide non visqueux, caractérisé à l'amont par un champ de vitesse composé d'une vitesse axiale uniforme $U_{0}$ et d'une rotation solide de vitesse angulaire $\Omega_{0}$, et tel que l'on ait partout $u_{z}>0$, les équations du mouvement se résument en une seule équation aux dérivées partielles linéaires sur la fonction de courant $\psi$, paramétrée par $S_{0}$ :

$$
\frac{\partial^{2} \psi}{\partial z^{2}}+\frac{1}{r} \frac{\partial}{\partial r}\left(\frac{1}{r} \frac{\partial \psi}{\partial r}\right)=2 S_{0}^{2} r^{2}-4 S_{0}^{2} \psi
$$

où $\psi$ est telle que

$$
u_{r}=-\frac{1}{r} \frac{\partial \psi}{\partial z} \quad \text { et } \quad u_{z}=\frac{1}{r} \frac{\partial \psi}{\partial r}
$$

L'équation (7) peut donc décrire de façon satisfaisante notre écoulement expérimental en-dehors des couches limites, à condition que l'écoulement amont reste proche de la condition que nous venons de citer, et qu'aucune recirculation n'ait lieu.

La solution de (7) ne peut être connue analytiquement que lorsque l'on suppose de plus l'écoulement parallèle (i.e. $\partial / \partial z=0)$. On peut donc à l'aide de ce modèle déterminer simplement la vitesse en sortie de tuyère convergente, mais pour le domaine entier une résolution numérique doit être mise en œuvre.

L'équation (7) étant elliptique, sa résolution implique la connaissance de conditions aux limites sur le bord du domaine entier. Celui-ci s'étend depuis la sortie du nid d'abeilles à l'amont, jusqu'au plan de sortie de la tuyère en aval. On impose donc les conditions suivantes :

- Section amont : $u_{z}=U_{0}$ et $u_{\theta}=r \Omega_{0}$

- Au centre : condition de symétrie $u_{\theta}=0$

- À la paroi : condition de glissement $u_{r}=0$

- Section de sortie : $u_{r}=0$.

Le problème est alors résolu à l'aide d'une méthode de collocation spectrale. 


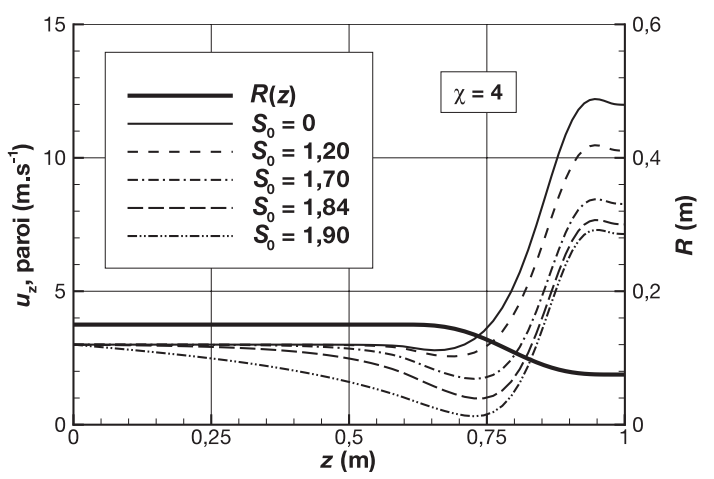

Fig. 4. Vitesse axiale à la paroi obtenue numériquement pour des valeurs croissantes de $S_{0}$. Tuyère de taux de contraction $\chi=4$.

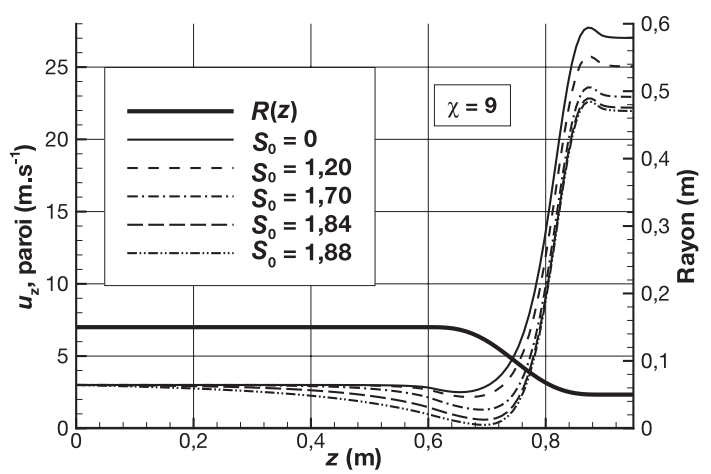

Fig. 5. Vitesse axiale à la paroi obtenue numériquement pour des valeurs croissantes de $S_{0}$. Tuyère de taux de contraction $\chi=9$.

\subsection{Résultats}

On constate (voir les Figs. 4 et 5) que le modèle ne permet d'obtenir une solution stationnaire de l'écoulement que jusqu'à une valeur limite $S_{0} \approx 1,88-1,90$. Au-delà, une recirculation intervient à la paroi de la conduite, en entrée de convergent, pour les deux taux de contractions étudiés. Tout se passe comme si la faible décélération qui se produit à la paroi en entrée de convergent en l'absence de rotation s'amplifiait progressivement, tant en amplitude qu'en extension spatiale.

Ce comportement peut être relié à un phénomène de transition critique comparable au passage de la limite sonique dans le cas d'un fluide compressible. En effet, les écoulements tournants sont le siège d'ondes de Kelvin (aussi appelées ondes d'inertie) dues à la force de Coriolis, pour lesquelles une vitesse de phase et une vitesse de groupe peuvent être définies de façon traditionnelle (voir [4] à titre d'exemple). En considérant un champ de vitesse local $\left(u_{r}(r), u_{\theta}(r), u_{z}(r)\right)$, on peut alors distinguer le régime supercritique, dans lequel ces ondes se propagent uniquement de l'amont vers l'aval, du régime subcritique dans lequel l'information peut remonter vers l'amont; à la transition, les ondes susceptibles de se propager vers l'amont correspondent à la limite des très grandes longueurs d'onde.
Si l'on considère à présent l'écoulement imposé à la station amont dans notre modèle, i.e. $\left(u_{r}(r)=0, u_{\theta}(r)=\right.$ $\left.r \Omega_{0}, u_{z}(r)=U_{0}\right)$, on constate que celui-ci est supercritique aux basses rotations, et que la transition critique s'effectue précisément pour $S_{0 \mathrm{c}} \approx 1,916$. À mesure que l'on se rapproche de ce régime, la «perturbation » due à l'entrée du fluide dans le convergent s'étend progressivement dans la portion de conduite de section constante et tend à se rapprocher de la section d'entrée. Ainsi que nous l'avons déjà signalé, la formulation du problème stationnaire est alors mise en défaut pour des valeurs supérieures du nombre de Swirl. Dans ces régimes subcritiques, l'apparition d'une bulle d'éclatement tourbillonnaire est possible (voir à cet effet la revue de Leibovich [5]), et toute perturbation située en aval, donc en particulier un convergent, est susceptible de modifier de façon significative l'écoulement [6].

L'éventualité d'une recirculation à la paroi d'un convergent abritant un écoulement défini par une condition amont de rotation solide et d'advection constante a déjà été évoquée par Batchelor [3]. En utilisant la formule analytique évoquée à la section 3.1, celui-ci a prédit la possibilité d'atteindre une vitesse nulle à la paroi dans le plan de sortie du convergent. Cependant celle-ci se produit pour un nombre de Swirl $S_{0 \mathrm{~B}}$ supérieur à la valeur limite $S_{0 \mathrm{c}}$ correspondant à la criticité de l'écoulement amont. Il est donc très probable que pour $S_{0}=S_{0 \mathrm{~B}}$, l'écoulement amont, alors subcritique, ne vérifie plus les hypothèses permettant d'arriver au résultat d'une recirculation dans le plan de sortie.

Plus récemment, Rusak et Meder [7] ont étudié à l'aide d'une analyse asymptotique l'influence d'une contraction de taux infinitésimal sur un écoulement amont plus général (de type q-vortex) proche du régime critique. Ils ont alors montré la possibilité d'une recirculation à la paroi, dans le plan de sortie. Nous pensons que cette localisation particulière de la recirculation en sortie provient du caractère infinitésimal de la contraction, qui permet d'obtenir un écoulement proche de la criticité dans toute la contraction. Ceci n'est pas le cas dans notre dispositif, puisqu'une contraction de taux « fini » comme la nôtre éloigne l'écoulement du régime critique en sortie.

\section{Calculs de stabilité}

\subsection{Stabilité globale}

Dans le cadre de travaux sur l'éclatement tourbillonnaire, Wang et Rusak [8] se sont intéressés au cas d'un écoulement tournant uniforme se développant dans une portion de conduite de section constante. Ils ont montré que le passage du régime critique s'accompagne de l'apparition d'une instabilité globale axisymétrique dans l'écoulement : une perturbation de grande extension spatiale s'amplifie si bien que la solution uniforme disparaît au profit d'une solution comportant un éclatement tourbillonnaire. L'objet de cette section est d'étudier dans notre cas la stabilité globale axisymétrique des 


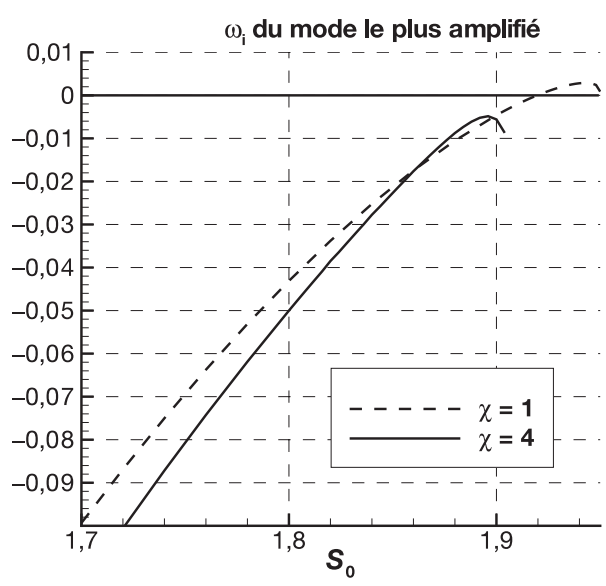

Fig. 6. Taux d'amplification $\omega_{i}$ du mode global le plus amplifié trouvé dans le dispositif comportant la tuyère $\chi=4$, en fonction de $S_{0}$. À titre de comparaison, la courbe $\chi=1$ correspond à un tuyau de section constante et de même longueur totale.

écoulements stationnaires décrits précédemment; en particulier, si certaines des solutions stationnaires sont globalement instables, celles-ci ne seront probablement pas observables expérimentalement.

On utilise donc une formulation instationnaire analogue à celle de l'équation (7), où intervient une variable supplémentaire, la circulation $K(r, z, t)=r u_{\theta}(r, z, t)$ (pour plus de détails voir [8]). L'analyse de stabilité globale consiste à superposer à l'écoulement stationnaire $\left\{\Psi_{0}(r, z), K_{0}(r, z)\right\}$, considéré comme écoulement moyen, une perturbation infinitésimale axisymétrique que l'on cherche sous la forme $\mathrm{e}^{-\mathrm{i} \omega t}\left\{\psi^{\prime}(r, z), K^{\prime}(r, z)\right\}$. L'écoulement est alors globalement instable lorsque $\operatorname{Im}(\omega)=\omega_{i}>0$, globalement stable sinon.

Nous trouvons que les écoulements présentés à la section 3 sont globalement stables aux perturbations axisymétriques, comme l'illustre la figure 6 pour le cas de la tuyère de taux de contraction $\chi=4$. La présence d'une tuyère convergente modifie donc l'écoulement stationnaire, mais ne change pas ses propriétés de stabilité globale. Ce résultat concorde également avec celui obtenu par Rusak et Meder [7] dans leur étude asymptotique pour une contraction de très faible amplitude.

\subsection{Amplification des perturbations à petite échelle}

Nous cherchons ici à savoir si une perturbation de très petite dimension caractéristique initiée à l'origine amont de l'écoulement est amplifiée ou atténuée le long de sa trajectoire dans l'écoulement stationnaire simulé à la section 3. Comme l'étude classique de stabilité locale ne peut être appliquée pour un écoulement fortement nonparallèle comme le nôtre, nous utilisons ici une analyse proche de la théorie de la distorsion rapide, faisant appel à un formalisme WKB (pour de plus amples détails voir [9] ou, à titre d'exemple, [10]).

La figure 7 représente l'amplification subie par une perturbation prenant naissance à la section amont, au

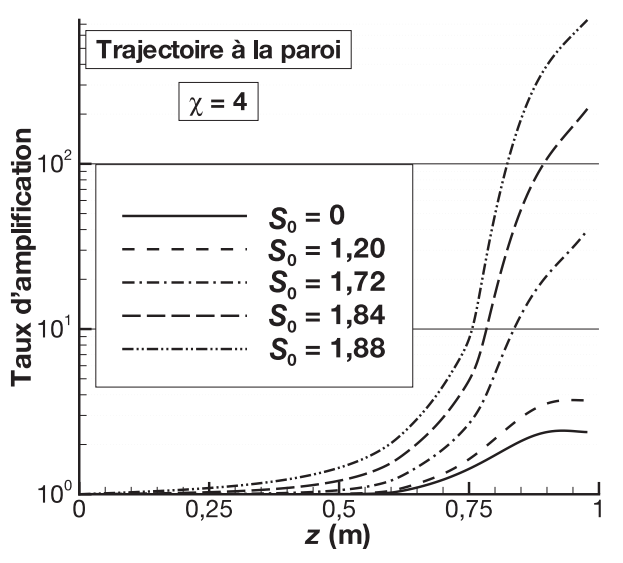

Fig. 7. Évolution du taux d'amplification d'une perturbation de très petite échelle initiée à l'origine amont de l'écoulement, à la paroi, pour différents $S_{0}$. Tuyère de taux $\chi=4$.

niveau de la paroi, et évoluant dans l'écoulement généré à l'aide de la tuyère de taux de contraction $\chi=4$. On constate qu'à l'approche de la limite critique, cette amplification subit une très forte augmentation, puisque le taux d'amplification est environ 300 fois plus élevé à $S_{0}=1,88$ qu'à $S_{0}=0$ (pour le convergent de taux $\chi=9$, on trouve un rapport d'environ 160). Il est à noter qu'en fait, plus la trajectoire considérée s'éloigne du centre, plus cette amplification est importante : le phénomène est donc maximal pour la trajectoire à la paroi, que nous avons représentée ici.

\section{Confrontation modèle-expérience}

La comparaison du modèle présenté dans les sections 3 et 4 avec l'écoulement expérimental appelle principalement deux remarques.

Tout d'abord, l'équation (7) suppose (notamment) que l'on ait $u_{z}=U_{0}$ à l'amont, quel que soit le nombre de Swirl $S_{0}$ pour lequel la solution existe. Du point de vue expérimental, on peut s'attendre à ce que la vitesse axiale en sortie du nid d'abeilles s'écarte progressivement de cette condition à mesure que $S_{0}$ augmente. En effet, le nid tournant de plus en plus vite, il est peu probable que la vitesse axiale du fluide entrant dans les alvéoles centrales, qui ont une vitesse de rotation très faible, soit la même que dans les alvéoles externes qui tournent le plus vite. Une exploitation plus poussée des profils expérimentaux dans le plan de sortie (que nous ne détaillerons pas ici) montre que le profil amont reste très proche des conditions théoriques jusqu'à $S_{0} \approx 1,20$, mais qu'il s'en écarte en effet progressivement pour des valeurs supérieures. À ce jour, cet écart n'est pas encore quantifié car nous ne disposons pas de mesures précises de l'écoulement en amont du convergent; celles-ci vont être réalisées prochainement (voir la Sect. 6). Néanmoins, nous pensons que cet écart est suffisamment faible pour que le scénario dynamique du modèle reste applicable à l'expérience. Le résultat de la section 4.2 montre que pour l'écoulement stationnaire théorique, les perturbations de petite échelle transportées 
sur des trajectoires suffisamment éloignées de l'axe de symétrie « explosent» lorsque les conditions en amont du convergent se rapprochent de la criticité, c'est-à-dire lorsque $1,70 \leq S_{0} \leq 1,90$. Ce résultat est en bon accord avec les mesures de la figure 3 : on constate que sur la trajectoire $r / R_{1}=0,6$, qui se situe en-dehors de la couche limite, le taux de turbulence azimutale commence à augmenter de façon significative pour des valeurs comparables. Ainsi, même si l'écoulement amont n'est plus rigoureusement proche du modèle théorique, l'étude de stabilité à petite échelle semble modéliser de façon réaliste l'apparition de la turbulence observée expérimentalement.

Par ailleurs, le modèle présenté concerne un écoulement de fluide parfait et n'aborde donc pas la question de la couche limite, qui rend le problème plus complexe. En effet, les vitesses axiales obtenues à la paroi et représentées aux figures 4 et 5 incitent à chercher la présence dans l'écoulement expérimental d'un décollement de couche limite. À ce jour, seules des visualisations qualitatives à l'aide de fils de cotons ( tufts») fixés à la paroi ont été mises en œuvre et n'ont pas permis de conclure à la présence d'un décollement. De plus, il faut signaler qu'en raison de la rotation et de la géométrie cylindrique de la veine, la couche limite de l'écoulement (qui est turbulente) se développe sur une paroi à courbure concave. Or plusieurs études ont montré que cette courbure particulière pouvait augmenter l'énergie cinétique turbulente de la couche limite ou même être à l'origine de l'apparition dans celle-ci de structures cohérentes (voir par exemple l'étude expérimentale de Barlow et al. [11]). L'influence de ces phénomènes sur l'écoulement global n'est sans doute pas négligeable, mais cette question dépasse le cadre de notre étude.

\section{Conclusion}

L'écoulement dans le plan de sortie d'un jet tournant généré à l'aide d'une tuyère convergente devient progressivement turbulent à mesure que le nombre de Swirl amont $S_{0}$ augmente. Cette évolution est similaire pour les deux convergents testés, qui possèdent des taux de contraction différents, si bien que l'explication de ce phénomène doit résider dans l'écoulement en amont du plan de sortie.

Sur le plan théorique, un modèle simple de fluide parfait axisymétrique avec une condition amont d'avection uniforme et de rotation solide permet de mettre en évidence la possibilité d'une recirculation à la paroi en entrée de convergent lorsque la limite critique (au sens des ondes d'inertie) est approchée. Les écoulements stationnaires correspondant à ce régime restent globalement stables aux perturbations axisymétriques mais amplifient les perturbations de petite échelle de façon beaucoup plus intense qu'un écoulement sans rotation.
En dehors de la région de proche paroi, l'écoulement expérimental reste très proche du modèle stationnaire tant que $S_{0} \leq 1,20$. Au-delà de cette valeur, la condition amont expérimentale commence à s'écarter de celle du modèle mais celui-ci parvient à rendre compte de l'apparition de turbulence observée en sortie de convergent, jusqu'à ce que la limite critique soit atteinte dans l'écoulement amont.

Il nous semble tout de même nécessaire d'affiner ce modèle de façon à prendre en compte une condition amont plus réaliste, ce qui permettrait également de déterminer si la phénoménologie théorique de recirculation à l'approche du régime critique reste valable. Sur le plan expérimental, nous avons l'intention de réaliser des visualisations plus complètes utilisant la PIV tridimensionnelle pour explorer l'écoulement directement en amont des tuyères convergentes. Nous espérons ainsi mieux connaître la condition amont à rotation élevée et confirmer ou infirmer l'hypothèse d'un décollement de couche limite ou d'une recirculation de plus grande amplitude, voire d'un éclatement tourbillonnaire.

\section{Références}

[1] S. Farokhi, R. Taghavi, Effect of initial swirl distribution on the evolution of a turbulent jet, AIAA Journal 27 (1988) 700-706

[2] P. Billant, J.-M. Chomaz, P. Huerre, Experimental study of vortex breakdown in swirling jets, J. Fluid Mech. 376 (1998) 183-219

[3] G.K. Batchelor, An Introduction to Fluid Dynamics, Cambridge University Press, 1967

[4] F. Gallaire, J.-M. Chomaz, The role of boundary conditions in a simple model of incipient vortex breakdown, Phys. Fluids 16 (2004) 274-286

[5] S. Leibovich, Vortex stability and breakdown : survey and extension, AIAA Journal 22 (1983) 1192-1206

[6] M.P. Escudier, J.J. Keller, Recirculation in swirling flow: a manifestation of vortex breakdown, AIAA Journal 23 (1985) 111-116

[7] Z. Rusak, C.C. Meder, Near-critical swirling flow in a slightly contracting pipe, AIAA Journal 42 (2004) 2284-2293

[8] S. Wang, Z. Rusak, The dynamics of a swirling flow in a pipe and transition to axisymmetric vortex breakdown, J. Fluid Mech. 340 (1997) 177-223

[9] A. Lifschitz, E. Hameiri, Local stability conditions in fluid dynamics, Phys. Fluids A 3 (1991) 2644-2651

[10] D. Sipp, L. Jacquin, Elliptic instability in twodimensional flattened taylor-green vortices, Phys. Fluids 10 (1998) 839-849

[11] R.S. Barlow, J.P. Johnston, Structure of a turbulent boundary layer on a concave surface, J. Fluid Mech. 191 (1988) 137-176 